\title{
Pelizaeus-Merzbacher-like disease
}

INSERM

\section{Source}

INSERM. (1999). Orphanet: an online rare disease and orphan drug data base. PelizaeusMerzbacher-like disease. ORPHA:280270

Pelizaeus-Merzbacher like disease (PMLD) is an autosomal recessive leukodystrophy sharing identical clinical and radiological features as X-linked Pelizaeus-Merzbacher disease (PMD; see this term). 